\title{
A Geometric Comparison of Single Chain Multi-State Models of Ion Channel Gating
}

Postprint of article published in: Bull. Math. Biol. 70 (5) (2008) 1503-1524.

\author{
Allan R. Willms ${ }^{1}$ \\ Dept. of Mathematics and Statistics \\ University of Guelph \\ Guelph, ON N1G 2W1 \\ Canada \\ ph: 519-824-4120 x52736 \\ fax: 519-837-0221 \\ AWillms@uoguelph.ca \\ Dominic Nelson ${ }^{2}$ \\ Dept. of Mathematics and Statistics \\ University of Guelph \\ Guelph, ON N1G 2W1 \\ Canada
}

May 30, 2008

${ }^{1}$ Corresponding Author
${ }^{2}$ Undergraduate student 


\begin{abstract}
Multi-state models of ion channel gating have been used extensively, but choosing optimally small yet sufficiently complex models to describe particular experimental data remains a difficult task. In order to provide some insight into appropriate model selection, this paper presents some basic results about the behaviour of solutions of multi-state models, particularly those arranged in a chain formation. Some properties of the eigenvalues and eigenvectors of constant-rate multi-state models are presented. A geometric description of a three-state chain is given and, in particular, differences between a chain equivalent to an Hodgkin-Huxley model and a chain with identical rates are analyzed. One distinguishing feature between these two types of systems is that decay from the open state in the HodgkinHuxley model is dominated by the most negative eigenvalue while the identical rate chain displays a mix of modes over all eigenvalues.
\end{abstract}

Keywords: Multi-state models, Ion channels, Geometric description, Hodgkin-Huxley model, Identical rate chain 


\section{Introduction}

Ion channels are proteins embedded in the membranes of neurons and muscle cells. They form a gateable pore which can open to allow the passage of ions, and hence electric charges in or out of the cell. Many channels are selective to the particular ions they allow to pass, and most open or close in response to external stimuli such as the binding of ligands or the electric field across the cell membrane. Ion channels have received a considerable amount of mathematical treatment since Hodgkin and Huxley (1952) first published their model of sodium and potassium conductance across the giant axon of Loligo. Hodgkin and Huxley modelled the potassium conductance as proportional to $x^{4}$ where $x$ obeyed a firstorder differential equation and represented the probability of a "gating charge" moving into position to open the channel. The power four (or, in the case of their sodium conductance, three) was chosen because it accounted for the observed delay in the increase of the current upon depolarization and as such was a "useful simplification" compared to higher-order equations (Hodgkin and Huxley, 1952, p. 506). In terms of the "gate" analogy, the power four represents four independent gates which must all open (or four gating particles which must all dock) for the channel to conduct ions.

Since 1952, the physical view of the ion channel has moved from independent gates which open and close to discrete states through which the channel protein progresses as it moves to a conformation where its pore is open to conduct ions. Mathematical models of ion channels have, therefore, moved from single first-order Hodgkin-Huxley (HH) type equations to systems of first-order differential equations where each variable represents the probability of being in a specific conformational state. (Of course, an $n^{\text {th }}$ order equation is equivalent to a system of $n$ first order equations, so this type of model was at least contemplated by Hodgkin and Huxley themselves.) Such models are called multi-state models, or Markov models since the future state of the channel depends only on the current state, and are represented by systems of linear equations. Typically, only one state is considered to be open, but models with multiple open states, or states with differing conductance levels are also possible. As has been pointed out by others and as we describe below, the Hodgkin-Huxley model can be viewed as a particular multi-state model, and so multi-state models are a generalization of their original model.

Transitions between states of a multi-state model are governed by rate functions, which for voltage-dependent ion channels, are functions of the voltage difference across the membrane. Such models can also be used for ligand-dependent channels (Destexhe et al., 1994). Thermodynamic arguments (Eyring et al., 1949; Tsien and Noble, 1969; Hill and Chen, 1972; Hille, 1992) relate the transition between states to either a charge surmounting a free-energy barrier, or rotation of a rigid dipole. In the simplest case where the free energy depends linearly on the voltage, they predict that the functional forms for the transitions back and forth between two states are simple exponentials (given below in Sect. 3) which depend on four independent parameters. More parameters arise if more complicated voltage-dependence relations are used (Destexhe and Huguenard, 2000). Thus, even if every new state is only connected to one other state, every new state added to the model introduces at least four new parameters. 
A consequence of using multi-state models is that even with a relatively small number of states, the number of free parameters is very large. On the one hand, this means that with the proper choice of parameters and connections between states, the model can replicate nearly any kinetic observations. On the other hand, the problem of determining an appropriately small model and the optimal parameter values for that model to best fit a given set of data is very difficult. This problem is compounded by a lack of information. Measurements of ionic conductance through the channel only provide direct information about the open state or states. The measurement of "gating currents" provides additional information on parameter values and on the dynamics among closed states, but not to the point of providing a time series for each state.

Operationally, the primary means of dealing with this excess of freedom in the models has been to restrict state connections and the rate functions in some manner. Typically, the states are ordered in a partial sequence with each state connected to usually two, sometimes three, or at most four others. The rate functions are often restricted by dictating that all or a set of the forward rates be identical or integer multiples of each other, and the reverse rates similarly (Zagotta et al., 1994b; Roux et al., 1998; Bezanilla, 2000; Bähring et al., 2001; Chanda and Bezanilla, 2002; Piper et al., 2003). Aggregation of similarly behaving states can also be performed to yield some simplification (Kienker, 1989; Kijima and Kijima, 1997).

Multi-state models are not the only type of model for ion channels. The large number of free parameters associated with multi-state models motivated the development of continuum models (Levitt, 1989). The technical advance of single-channel recordings has resulted in fractal models championed by Liebovitch and others (Liebovitch et al., 1987; Liebovitch and Todorov, 1996; Liebovitch et al., 2001). A review of various stochastic models for ion channels can be found in Ball and Rice (1992). Nonetheless, multi-state models continue to enjoy considerable use.

This paper presents some basic results about the behaviour of solutions of multi-state models, particularly those whose states are arranged in a chain formation, and provides a geometric comparison between a Hodgkin-Huxley type model and other multi-state models. The motivation is to provide insight into some of the properties of these models and consequently aid in the selection of appropriate models for particular channels.

In Sect. 2, we present some basic results about multi-state models of ion channels regarding their equilibria and eigenvalues, and we specialize these results to single chain systems including a chain equivalent to the $\mathrm{HH}$ model and a chain with identical forward and backward rates. In Sect. 3, we give a geometric description of a three-state model and particularly compare the behaviour of the $\mathrm{HH}$ chain with the identical rate chain. Sect. 4 provides some discussion.

\section{Equilibria and Eigenvalues of Multi-State Models}

We are interested in models of ion channel gating where the model states represent different physical conformations of the channel protein as it transitions between a non-conducting (closed pore) and conducting (open pore) state. Typically, although not necessarily, one state, is regarded as the open state, and the remaining states are considered closed. Models 
could also allow several open states with possibly differing conductance levels. For voltagegated ion channels, the rates of transition between states depend on the voltage difference across the neuronal membrane. For ligand-gated channels, the rates would depend on the abundance of ligand. When the transition rates are constant (experimentally a voltage-clamp situation for voltage-gated channels, or a constant concentration of ligand for ligand-gated channels), the models become systems of constant coefficient linear differential equations and are thus amenable to considerable analysis. In this section, we describe how the model forms and physical constraints dictate the qualitative behaviour of these models, namely, the system has a unique asymptotically stable equilibrium point and all of the eigenvalues are real. Although these results are generally known and follow from basic analysis, we provide them here for completeness and clarity.

We model the transitions between states as a simple continuous-time Markov process with $n+1$ states where $x_{i}(t)$ is the probability of being in state $i$ at time $t, i=0, \ldots, n$, and $\rho_{i j}$ is the rate at which a channel in state $i$ moves to state $j$. Let $R$ be the matrix whose $i$ th row $j$ th column entry is $\rho_{i j}$. The governing system is

$$
x^{\prime}=A x,
$$

with

$$
A=R^{T}-\operatorname{diag}(R \mathbf{1})
$$

where $\mathbf{1}$ is the column vector of ones in $\mathbb{R}^{n+1}$, and $\operatorname{diag}(v)$ is the diagonal matrix constructed from the entries of the vector $v$.

The model structure immediately constrains the dynamics. The form of $A$ given in (2) implies that $\mathbf{1}^{T} A=0$, that is, the entries in each column add to zero, thus $\left(\mathbf{1}^{T} x\right)^{\prime}=0$ and, therefore, $\mathbf{1}^{T} x$ is a constant for all time. We require the probabilities of being in each state to add to one, hence we are interested in dynamics on the particular invariant manifold

$$
M=\left\{x \in \mathbb{R}^{n+1} \mid \mathbf{1}^{T} x=1\right\} .
$$

In addition, the physical situation imposes several restrictions on the transition rates which in turn constrain the dynamics. First, $\rho_{i j} \geq 0$ and $\rho_{i i}=0$. (From the form of $A$, this immediately implies that the non-negative orthant for (1) is invariant.) Secondly, we assume every subset of states leads to at least one other state, in other words, no subset of states is absorptive, and the system does not decompose into two or more disjoint subsystems. Mathematically this assumption is: for each non-empty proper subset $P$ of $\{0, \ldots, n\}$ not all $\rho_{i j}, i \in P, j \notin P$ can be zero. This assumption implies, in the case of constant rates, uniqueness of an equilibrium on $M$ in the positive orthant as the following lemma and theorem show.

Lemma 1 The null space of $A$ is spanned by a vector whose entries are all positive.

Proof: Since $\mathbf{1}^{T} A=0$, the null space of $A$ must have dimension at least one. Let $x \neq 0$ be in the null space of $A$ and suppose there is a non-empty proper subset $P$ of $\{0, \ldots, n\}$ such 
that $x_{i}$ is positive if and only if $i \in P$. Let $S$ be the selection vector for $P$, that is, $S_{i}=1$ if $i \in P$ and $S_{i}=0$ if $i \notin P$. Consider $S^{T} A x$. Using (2) and the fact that $A x=0$, we have

$$
\begin{aligned}
S^{T} R^{T} x & =S^{T} \operatorname{diag}(R \mathbf{1}) x \\
\sum_{j \in P} \sum_{i=0}^{n} \rho_{i j} x_{i} & =\sum_{i \in P} \sum_{j=0}^{n} \rho_{i j} x_{i} \\
\sum_{j \in P} \sum_{i \notin P} \rho_{i j} x_{i} & =\sum_{i \in P} \sum_{j \notin P} \rho_{i j} x_{i}
\end{aligned}
$$

Clearly the left side of the above expression is non-positive while the right side is nonnegative. Hence equality requires both sides to be zero, but since $x_{i}>0$ for $i \in P$, the only way the right side can be zero is if $\rho_{i j}=0$ for all $i \in P$ and all $j \notin P$ which violates the second assumption above. We conclude that any non-zero vector $x$ in the null space of $A$ must either have all entries positive, or all entries non-positive. No two or higher dimensional subspace of $\mathbb{R}^{n+1}$ is contained in this region and every one dimensional subspace contained in it necessarily enters the positive orthant. Hence, there must be a vector all of whose entries are positive which spans the null space of $A$.

Theorem 1 There is a unique vector $x^{*}$ satisfying $A x^{*}=0$ and $\mathbf{1}^{T} x^{*}=1$. Furthermore, all entries of $x^{*}$ are positive.

Proof: By the previous lemma, there is a vector $w$, whose entries are all positive, that is a basis for the null space of $A$. Since $A x^{*}=0, x^{*}=c w$ for some real number $c$. But $\mathbf{1}^{T} c w=1$ has the unique solution $c=1 / \sum_{i=0}^{n} w_{i}$ which is clearly positive, hence all entries of $x^{*}$ are positive.

Finally, as a last assumption, we require that at thermodynamic equilibrium there is no net flow between any two states. This prevents there being a net directional movement of channels at equilibrium around a set of states connected in a loop. This latter assumption is called the law of detailed balance and it may be expressed as

$$
\rho_{i j} x_{i}^{*}=\rho_{j i} x_{j}^{*}, \quad \forall i, j \in\{0, \ldots, n\}
$$

or equivalently,

$$
\operatorname{diag}\left(x^{*}\right) R=R^{T} \operatorname{diag}\left(x^{*}\right)
$$

(Fredkin et al., 1985). This final assumption constrains the eigenvalues of $A$ :

Lemma 2 (Fredkin et al., 1985, p. 277) A is real diagonalizable.

Proof: As a consequence of (4), from (2), we have

$$
\operatorname{Adiag}\left(x^{*}\right)=\operatorname{diag}\left(x^{*}\right) A^{T} .
$$


Since all entries of $x^{*}$ are positive, we may define the symmetric, invertible matrix $W=$ $\operatorname{diag}\left(\sqrt{x^{*}}\right)$ and thus, multiplying the above by $W^{-1}$ on both the left and the right gives

$$
W^{-1} A W=W A^{T} W^{-1}=\left(W^{-1} A W\right)^{T} .
$$

Therefore $A$ is similar to a real symmetric matrix and thus $A$ is real diagonalizable.

In particular then, $A$ never has complex eigenvalues. The model form and physical constraints on the transition rates also imply that $n$ of the real eigenvalues are negative:

Theorem 2 One eigenvalue of $A$ is zero and the remaining $n$ satisfy $-2 \max _{i} \sum_{j=0}^{n} \rho_{i j} \leq$ $\lambda<0$.

Proof: We have already shown that the null space of $A$ has dimension one, hence exactly one eigenvalue is zero. Lemma 2 also dictates that the remaining eigenvalues are also real. By a well-known theorem on eigenvalue estimates (Mirsky, 1963, p. 212), all eigenvalues $\lambda$ of $A=\left[a_{i j}\right]$ must satisfy one of the inequalities

$$
\left|\lambda-a_{i i}\right| \leq \sum_{j \neq i}\left|a_{j i}\right|, \quad \text { for some } \mathrm{i}=0, \ldots, n
$$

But by construction of $A$ and since $\rho_{i j} \geq 0$ and $\rho_{i i}=0$, we have $\sum_{j \neq i}\left|a_{j i}\right|=\left|a_{i i}\right|$, and $a_{i i}=-\sum_{j=0}^{n} \rho_{i j}<0$. Thus all but the single zero eigenvalue of $A$ satisfy

$$
-2 \sum_{j=0}^{n} \rho_{i j} \leq \lambda<0, \quad \text { for some } i=0, \ldots, n
$$

Furthermore, let $\lambda_{0}=0$ and denote the non-zero eigenvalues of $A$ as $\lambda_{i}, i=1, \ldots, n$. Besides the bounds on the eigenvalues provided by (5), by computing the trace of $A$, we have

$$
\sum_{i=1}^{n} \lambda_{i}=-\sum_{i=0}^{n} \sum_{j=0}^{n} \rho_{i j}
$$

The preceding results allow us to fully describe the qualitative dynamics on $M$ for (1), in the case where rates $\rho_{i j}$ are constants. By Theorem $1, x^{*}$ is a unique fixed point on $M$. If we let $y=x-x^{*}$, the manifold $M$ is given by $\mathbf{1}^{T} y=0$. Since $\mathbf{1}$ is orthogonal to all vectors in $M$ and since it is a left eigenvector of $A$ corresponding to the zero eigenvalue $\left(\mathbf{1}^{T} A=0\right)$, we conclude that the $n$-dimensional manifold $M$ is precisely the eigenspace of $x^{*}$ corresponding to the $n$ negative eigenvalues of $A$ given by Theorem 2. Consequently, for this linear system restricted to $M, x^{*}$ is globally asymptotically stable, and all of the eigenvalues are real and negative. 


\subsection{Single Chain Models}

States connected sequentially in a straight line are commonly employed as models or parts of models of ion channel gating. In this case, each state save the end two, is connected to two other states to form a single chain of states. Setting $\rho_{i, i+1}=r_{i}$ and $\rho_{i+1, i}=l_{i+1}$, $i=0, \ldots, n-1$, the model is

$$
x_{0} \underset{l_{1}}{\stackrel{r_{1}}{\rightleftarrows}} x_{1} \underset{l_{2}}{\stackrel{r_{2}}{\rightleftarrows}} x_{2} \cdots x_{n-1} \underset{l_{n}}{\stackrel{r_{n}}{\rightleftarrows}} x_{n}
$$

We call such models "single chain multi-state models" or simply "single chain models." The matrix $A(2)$ is thus tri-diagonal:

$$
A=\left(\begin{array}{cccccc}
-r_{1} & l_{1} & & & & \\
r_{1} & -r_{2}-l_{1} & l_{2} & & 0 & \\
& r_{2} & -r_{3}-l_{2} & \ddots & & \\
& & r_{3} & \ddots & l_{n-1} & \\
& 0 & & \ddots & -r_{n}-l_{n-1} & l_{n} \\
& & & & r_{n} & -l_{n}
\end{array}\right)
$$

Defining $r_{n+1}$ and $l_{0}$ to be zero, the result of Theorem 2 in this case can be expressed as

$$
\lambda_{0}=0, \quad-2 \max _{0 \leq i \leq n}\left(r_{i+1}+l_{i}\right) \leq \lambda_{j}<0, \quad 1 \leq j \leq n,
$$

and (6) becomes

$$
\sum_{i=1}^{n} \lambda_{i}=-\sum_{i=1}^{n}\left(r_{i}+l_{i}\right)
$$

For this tri-diagonal system, we can also obtain a simple expression for the product of the $n$ non-zero eigenvalues. Since there is one zero eigenvalue, the coefficient (call it $C_{1}$ ) of the first order term in the characteristic polynomial $\chi_{A}(\lambda)=|\lambda I-A|$ is equal to $(-1)^{n} \prod_{i=1}^{n} \lambda_{i}$. But $C_{1}$ is also equal $(-1)^{n}$ times the sum of all $n$-rowed principal minors of $A$ (Mirsky, 1963, p. 197). These minors are obtained by deleting the $i$ th row and column from $A$, for $i=0, \ldots, n$, and then taking the determinant of the resulting $n \times n$ matrix. Since $A$ is tri-diagonal each of these $(n \times n)$ submatrices is block diagonal with one (if $i=0$ or $i=n$ ) or two $(0<i<n)$ tridiagonal blocks. Let $A_{i, j}$ denote the submatrix of $A$ obtained by keeping only those entries in rows and columns $i$ through $j$ inclusive. If $j<i$, then $A_{i, j}$ is empty and define $\left|A_{i, j}\right|=1$. Then

$$
C_{1}=(-1)^{n} \prod_{i=1}^{n} \lambda_{i}=(-1)^{n} \sum_{i=0}^{n}\left|A_{0, i-1}\right|\left|A_{i+1, n}\right|,
$$

which can be simplified by the following lemma. 
Lemma 3 For A given by (8),

$$
\begin{aligned}
& \left|A_{0, i-1}\right|=(-1)^{i} \prod_{j=1}^{i} r_{j}, \quad i=1, \ldots, n, \\
& \text { and } \\
& \left|A_{i+1, n}\right|=(-1)^{n-i} \prod_{j=i+1}^{n} l_{j}, \quad i=0, \ldots, n-1 .
\end{aligned}
$$

Proof: For $A_{0, i-1}$, consecutively applying the row operations "add row $j$ to row $j+1$ " for $j=$ $0, \ldots, i-2$ yields an upper triangular matrix with diagonal entries $-r_{j}, j=1, \ldots, i$. Since these row operations do not alter the value of the determinant, the result follows. Similarly, consecutively applying the row operations "add row $j$ to row $j-1$ " for $j=n, n-1, \ldots, i+2$ to $A_{i+1, n}$ yields a lower triangular matrix with diagonal entries $-l_{j}, j=i+1, \ldots, n$ whose determinant is as specified.

We can now establish:

Theorem 3 For A given by (8), the non-zero eigenvalues satisfy

$$
\prod_{i=1}^{n} \lambda_{i}=(-1)^{n} \sum_{i=0}^{n}\left[\prod_{j=1}^{i} r_{j}\right]\left[\prod_{j=i+1}^{n} l_{j}\right]
$$

where, by definition, we take an empty product to be one.

Proof: The result follows immediately from (11) and Lemma 3.

\subsubsection{Hodgkin-Huxley Type Chains}

Now consider a single chain multi-state model where the rates in (7) are given by the special form $r_{i}=(n-i+1) \alpha$, and $l_{i}=i \beta, i=1, \ldots, n$ :

$$
x_{0} \underset{\beta}{\stackrel{n \alpha}{\rightleftarrows}} x_{1} \underset{2 \beta}{\stackrel{(n-1) \alpha}{\rightleftarrows}} x_{2} \cdots x_{n-1} \underset{n \beta}{\stackrel{\alpha}{\rightleftarrows}} x_{n}
$$

The governing system of differential equations is

$$
x_{j}^{\prime}=(n-j+1) \alpha x_{j-1}-[(n-j) \alpha+j \beta] x_{j}+(j+1) \beta x_{j+1}, \quad j=0, \ldots, n,
$$

where $x_{-1}$ and $x_{n+1}$ are defined to be zero. By setting

$$
x_{j}=\left(\begin{array}{c}
n \\
j
\end{array}\right) m^{j}(1-m)^{n-j}
$$

it is clear that $\sum_{j=0}^{n} x_{j}=(m+(1-m))^{n}=1$. Further, substituting (15) into (14), a straight forward calculation shows that for each $j$, the differential equation becomes

$$
m^{\prime}=\alpha(1-m)-\beta m \text {. }
$$


This is the classical Hodgkin-Huxley differential equation for a gate of a voltage-gated ion channel. Here $m$ represents the probability of the gate being in the open state, and $\alpha$ and $\beta$ are the rates of opening and closing of the gates, respectively (both depending continuously on the voltage). If there are $n$ independent gates in the channel and if $\bar{g}$ is the maximal conductance of the channel (all gates fully open), then the conductance of the channel is given by $g=\bar{g} m^{n}$, which is equivalent to saying that $x_{n}$ is the open state in the single chain multi-state model (14), and all other states are closed. Hodgkin and Huxley used specific forms for how the rate functions depend on the voltage, but we will name any model of the form (14) as an Hodgkin-Huxley type chain, or simply HH chain.

The equivalence between these two models as described above was first noted by Fitzhugh (1965) although with some notational differences, and has been described by others, for example, Destexhe et al. (1994). Fitzhugh also noted that this equivalence is not dependent on the rates $(\alpha$ and $\beta$ ) being constants. But we stress that the equivalence is only valid if the initial condition for $x$ is of the form $x_{j}=\left(\begin{array}{c}n \\ j\end{array}\right) m_{0}^{j}\left(1-m_{0}\right)^{n-j}$ where $m_{0}$ is the initial value for $m$. Mathematically, this is a substantial restriction on the form of the initial condition for $x$, limiting it to a one-dimensional manifold in the $n$-dimensional manifold $M$. If this condition is not met, then solutions of (14) do not take the form (15) and clearly the $n$-dimensional Markov system on $M$ has much richer behaviour than the one-dimensional $\mathrm{HH}$ equation. Biologically, however, this restriction is not too severe. In the constant rate function (constant voltage) situation, we have established that the multi-state model has a unique globally attractive equilibrium point, $x^{*}$. Since (16) clearly has a unique equilibrium given by

$$
m_{\infty}=\frac{1}{1+\beta / \alpha}
$$

it immediately follows that $x^{*}$ is given by (15) with $m=m_{\infty}$. In other words, the equilibrium of the multi-state model lies on the one dimensional manifold of initial conditions for which the multi-state model has solutions given by (15). Consequently, if the voltage is roughly constant for a long enough time, the solution of the multi-state system will approach the manifold on which the solutions of the multi-state system are governed by the solutions of the single HH differential Eq. (16). If the ion channel's state can only be altered by variations in the voltage level, this situation will remain. However, if the ion channel can at sometimes alter its states in a manner where the Markov model need not hold, for example, a fluctuation in the state due to a mechanical stress, then the initial conditions may no longer lie on the equilibrium manifold.

In the case of constant rates, the eigenvalues for (14) are easily obtained. It is not difficult to show that the solution to (16) is

$$
m=m_{\infty}+\left(m_{0}-m_{\infty}\right) e^{-(\alpha+\beta) t} .
$$

From (15), it then immediately follows that the components of $x$ are linear combinations of terms of the form $e^{-i(\alpha+\beta) t}$, for $i=0, \ldots, n$. From the theory of linear constant coefficient differential equations, we are then able to conclude that the eigenvalues of $A$ must be

$$
\lambda_{i}=-i(\alpha+\beta), \quad i=0, \ldots, n .
$$


Therefore, the sum and product of the non-zero eigenvalues for the $\mathrm{HH}$ chain are:

$$
\begin{aligned}
& \sum_{i=1}^{n} \lambda_{i}=-\frac{(n+1) n}{2}(\alpha+\beta) \\
& \prod_{i=1}^{n} \lambda_{i}=(-1)^{n} n !(\alpha+\beta)^{n} .
\end{aligned}
$$

\subsubsection{Identical Rate Chain}

Another commonly employed single chain multi-state model is similar to the Hodgkin-Huxley model, but instead of the rates between consecutive states changing linearly, they are all set equal, that is, $r_{i}=\alpha$, and $l_{i}=\beta$ for $i=1, \ldots, n$ in (7).

$$
x_{0} \underset{\beta}{\stackrel{\alpha}{\rightleftarrows}} x_{1} \underset{\beta}{\stackrel{\alpha}{\rightleftarrows}} x_{2} \cdots x_{n-1} \underset{\beta}{\stackrel{\alpha}{\rightleftarrows}} x_{n}
$$

We call this an Identical Rate (IR) chain. The governing system of differential equations is

$$
x_{j}^{\prime}=\alpha x_{j-1}-[\alpha+\beta] x_{j}+\beta x_{j+1}, \quad j=0, \ldots, n,
$$

where $x_{-1}$ and $x_{n+1}$ are defined to be zero. Although this system appears at least as simple as (14), it does not, unlike the HH system, admit a simple solution dependent on a single differential equation. Yueh (2005) has recently obtained analytic expressions for the eigenvalues of a generalization of the above system. We can deduce from his work that the eigenvalues for the IR chain are

$$
\lambda_{0}=0, \quad \lambda_{i}=-(\alpha+\beta)+2 \sqrt{\alpha \beta} \cos \frac{i \pi}{(n+1)}, \quad 1 \leq i \leq n .
$$

Directly from these, or using (10) and (12), we have:

$$
\begin{gathered}
\sum_{i=1}^{n} \lambda_{i}=-n(\alpha+\beta) \\
\prod_{i=1}^{n} \lambda_{i}=(-1)^{n} \sum_{i=0}^{n} \alpha^{i} \beta^{n-i}= \begin{cases}(-1)^{n}(n+1) \alpha^{n}, & \alpha=\beta \\
(-1)^{n}\left(\frac{\alpha^{n+1}-\beta^{n+1}}{\alpha-\beta}\right), & \alpha \neq \beta\end{cases}
\end{gathered}
$$

\section{Geometrical Description of a Three-State Single Chain Model}

We consider now a three-state single chain model for a voltage-gated ion channel and provide a geometrical description of its solutions. Although most models employed for real channels have more than three states, the geometrical description we provide here (made amenable since the system restricted to the manifold $M$ is planar) gives some insight into these models 
and, in particular, to the differences between Hodgkin-Huxley models and more general multi-state models. The purpose is to identify distinguishing features of these two types of models. We compare the locations of equilibria of the two models, and the orientation of the eigenvectors at these equilibria. Primarily, we are concerned with solutions that start at one equilibrium point and then, due to a sudden voltage jump which alters the system, move to the equilibrium point of the system under the new voltage value. This represents the typical experimental voltage-clamp situation. In the three-state model, there are just two exponential rates of movement (two non-zero eigenvalues), and we compare the relative sizes of these two exponentials in solutions depending on the locations of the pre-step and post-step equilibrium points. Implications of the results of this section are discussed in Sect. 4.

The model for a three-state chain is given by (7) with $n=2$ and the governing equation is $x^{\prime}=A x$, where

$$
A=\left(\begin{array}{ccc}
-r_{1} & l_{1} & 0 \\
r_{1} & -r_{2}-l_{1} & l_{2} \\
0 & r_{2} & -l_{2}
\end{array}\right)
$$

Since the dynamics of interest are restricted to the manifold $M\left(x_{0}+x_{1}+x_{2}=1\right)$, it is simpler and sufficient to consider the projection of the system onto the $\left(x_{0}, x_{2}\right)$-plane. On this plane, the dynamics are bounded by the coordinate axes and the line $x_{0}+x_{2}=1$; denote this triangle as $\Delta$. The governing system on $\Delta$ is

$$
\left[\begin{array}{l}
x_{0} \\
x_{2}
\end{array}\right]^{\prime}=\left(\begin{array}{cc}
-r_{1}-l_{1} & -l_{1} \\
-r_{2} & -l_{2}-r_{2}
\end{array}\right)\left[\begin{array}{l}
x_{0} \\
x_{2}
\end{array}\right]+\left[\begin{array}{l}
l_{1} \\
r_{2}
\end{array}\right] .
$$

The rate functions are dependent on the voltage, $V$. If the voltage is constant, the system has a unique equilibrium point

$$
\begin{aligned}
& x_{0}=\left(\frac{l_{1}}{r_{1}}\right)\left(\frac{l_{1}}{r_{1}}+1+\frac{r_{2}}{l_{2}}\right)^{-1}=\frac{L}{L+1+R}, \\
& x_{2}=\left(\frac{r_{2}}{l_{2}}\right)\left(\frac{l_{1}}{r_{1}}+1+\frac{r_{2}}{l_{2}}\right)^{-1}=\frac{R}{L+1+R},
\end{aligned}
$$

where for convenience we have defined $L=l_{1} / r_{1}$ and $R=r_{2} / l_{2}$. At different constant voltage levels, the equilibrium will be at different locations in the triangle $\Delta$, forming a curve, $C(V)$, defined by the parametric Eqs. (26).

We restrict the rate functions by the following two assumptions.

Assumption 1 For fixed $\sigma$ equal to either +1 or -1 ,

$$
\lim _{V \rightarrow-\sigma \infty} L=\lim _{V \rightarrow \sigma \infty} R=\infty \quad \text { and } \quad \lim _{V \rightarrow \sigma \infty} L=\lim _{V \rightarrow-\sigma \infty} R=0 .
$$

This assumption dictates that at opposite extreme voltage levels, the equilibrium state is concentrated in opposite extreme ends of the chain. An activation gate which opens as $V$ increases corresponds to $\sigma=+1$, while an inactivation gate which opens as $V$ decreases corresponds to $\sigma=-1$. 


\section{Assumption 2}

$$
\frac{d R}{d V} \text { and } \quad \frac{d L}{d V} \quad \text { are non-zero and of opposite signs. }
$$

This assumption provides some monotonicity, in the sense that as the voltage level moves from one extreme to another, the equilibrium state moves monotonically away from one extreme end of the chain to the other.

These assumptions are significant and exclude possibilities such as state one being the preferred state at extremely high voltages, or the equilibrium value of state 2 having a nonmonotone increase with voltage. Nonetheless, most multi-state models of ion channels satisfy these assumptions.

Within these two assumptions, we further consider several special cases.

Case 1 The model is an Hodgkin-Huxley chain. Thus, $r_{1}=2 r_{2}=2 \alpha$ and $2 l_{1}=l_{2}=2 \beta$ which yields $L=1 /(4 R)=\beta /(2 \alpha)$.

Case 2 The model is an identical rate chain. Thus, $r_{1}=r_{2}=\alpha$ and $l_{1}=l_{2}=\beta$ which yields $L=1 / R=\beta / \alpha$.

Case 3 The rate functions are simple exponentials (the most most commonly employed functional form for voltage-dependent rates (Zagotta et al., 1994b; Chanda and Bezanilla, 2002; Kargol et al., 2002; Piper et al., 2003)) of the form

$$
\begin{aligned}
& r_{i}=\bar{r}_{i} \exp \left(z_{i} e_{0} \delta_{i} V / k T\right) \\
& l_{i}=\bar{l}_{i} \exp \left(-z_{i} e_{0}\left(1-\delta_{i}\right) V / k T\right), \quad i=1, \ldots, n
\end{aligned}
$$

where $k$ is the Boltzmann constant, $T$ is the absolute temperature, $V$ is the voltage of the inside relative to the outside of the membrane, $e_{0}$ is the elementary electric charge, $z_{i}$ is the number of positive charges shifted toward the outside of the membrane as the channel moves from state $i$ to state $i+1, \delta_{i}$ is a constant between zero and one which represents the relative location of the thermodynamic energy barrier between the two states, and $\bar{r}_{i}$ and $\bar{l}_{i}$ are constant rates of transition in the absence of an electric potential. In this case, $L=\frac{\bar{l}_{1}}{\bar{r}_{1}} \exp \left(-z_{1} e_{0} V / k T\right)$ and $R=\frac{\bar{r}_{2}}{l_{2}} \exp \left(z_{2} e_{0} V / k T\right)$.

It should be noted that while Case 1 and Case 2 are mutually exclusive, Case 3 may or may not co-exist with either of the first two. For Case 3, Assumptions 1 and 2 are met if and only if $z_{1}$ and $z_{2}$ are of the same sign. For Case 1 and Case 2, the assumptions would be satisfied, for example, if $\alpha$ is a strictly increasing function tending to 0 as $V \rightarrow-\infty$ and $\beta$ is a strictly decreasing function tending to 0 as $V \rightarrow \infty$. Switching these properties of $\alpha$ and $\beta$ would also satisfy these assumptions.

\subsection{Shape of the Equilibrium Curve}

What is the general shape of the equilibrium curve? Since $L$ and $R$ are necessarily positive, Assumption 1 implies that the equilibrium curve has end points at $\left(x_{0}, x_{2}\right)=(1,0)$ and $(0,1)$ 
reached as $V \rightarrow \pm \infty$. From (26), we have

$$
x_{0}^{\prime}=\frac{L^{\prime}(R+1)-L R^{\prime}}{(L+1+R)^{2}} \quad \text { and } \quad x_{2}^{\prime}=\frac{R^{\prime}(L+1)-R L^{\prime}}{(L+1+R)^{2}},
$$

where the prime denotes differentiation with respect to $V$. Now since by Assumption $2, L^{\prime}$ and $R^{\prime}$ have opposite signs, it immediately follows that the numerators of $x_{0}^{\prime}$ and $x_{2}^{\prime}$ are of opposite sign and are never zero. Therefore, $x_{0}$ and $x_{2}$ are invertible functions of $V$ and $x_{2}$ can also be expressed as a strictly decreasing function of $x_{0}$ with derivative

$$
\frac{d x_{2}}{d x_{0}}=\frac{x_{2}^{\prime}}{x_{0}^{\prime}}=\frac{R^{\prime}(L+1)-R L^{\prime}}{L^{\prime}(R+1)-L R^{\prime}} .
$$

The second derivative of the equilibrium curve, $C(V)$, is

$$
\frac{d^{2} x_{2}}{d x_{0}^{2}}=\frac{1}{x_{0}^{\prime}}\left(\frac{x_{2}^{\prime}}{x_{0}^{\prime}}\right)^{\prime}=\left(L^{\prime} R^{\prime \prime}-L^{\prime \prime} R^{\prime}\right)(L+1+R)^{3} /\left(L^{\prime}(R+1)-L R^{\prime}\right)^{3} .
$$

For either an HH type chain or an IR chain, we have that $L=b / R$ for some positive constant b. In these cases, (30) simplifies to

$$
\frac{d^{2} x_{2}}{d x_{0}^{2}}=\frac{2 R^{3}(b / R+1+R)^{6}}{(2 / R+1)^{3}}
$$

which is always positive. For Case 3,

$$
\begin{aligned}
\frac{L^{\prime} R^{\prime \prime}-L^{\prime \prime} R^{\prime}}{L^{\prime}(R+1)-L R^{\prime}} & =\frac{-q_{1} \frac{\bar{l}_{1}}{\bar{r}_{1}} e^{-q_{1} V} q_{2}^{2} \frac{\bar{r}_{2}}{\bar{l}_{2}} e^{q_{2} V}-q_{1}^{2} \frac{\bar{l}_{1}}{\bar{r}_{1}} e^{-q_{1} V} q_{2} \frac{\bar{r}_{2}}{\bar{l}_{2}} e^{q_{2} V}}{-q_{1} \bar{l}_{1} e^{-q_{1}} e^{-q_{1}}\left(\frac{\bar{r}_{2}}{l_{2}} e^{q_{2} V}+1\right)-q_{2} \frac{\bar{l}_{1} \bar{r}_{2}}{\bar{r}_{1} \bar{l}_{2}} e^{\left(q_{2}-q_{1}\right) V}} \\
& =\frac{q_{2}\left(q_{1}+q_{2}\right)}{\frac{\bar{l}_{2}}{\bar{r}_{2}} e^{-q_{2} V}+\left(\frac{q_{2}}{q_{1}}+1\right)},
\end{aligned}
$$

where $q_{i}=z_{i} e_{0} / k T$. Since it has already been established that Assumptions 1 and 2 require that $z_{1}$ and $z_{2}$ have the same sign, the second derivative is again necessarily positive. Thus in all cases, the equilibrium curve never has an inflection point.

For Hodgkin-Huxley type and identical rate chains, since $L$ and $R$ are inversely related, the equilibrium curve is completely dictated in its shape. These shapes are illustrated in Fig. 1A. In contrast, there is a great deal of freedom in the shape of the equilibrium curve if only Case 3 holds, since $L$ and $R$ are independent. Some of the possible shapes are shown in Fig. 1B. This figure illustrates that the steady states for an identical rate chain always have a smaller component of $x_{1}$, the intermediate state, than a Hodgkin-Huxley type chain. If the voltage is slowly altered so that the system moves from the extreme where $x_{0}=1$ to the extreme $x_{2}=1$, then between these two extremes a Hodgkin-Huxley type chain will accumulate more in the $x_{1}$ state than an identical rate chain. The straight dotted line in Fig. 1 represents $x_{1}=0$; the closer the equilibrium curve is to this line, the more the system behaves as if there was no intermediate state at all. 

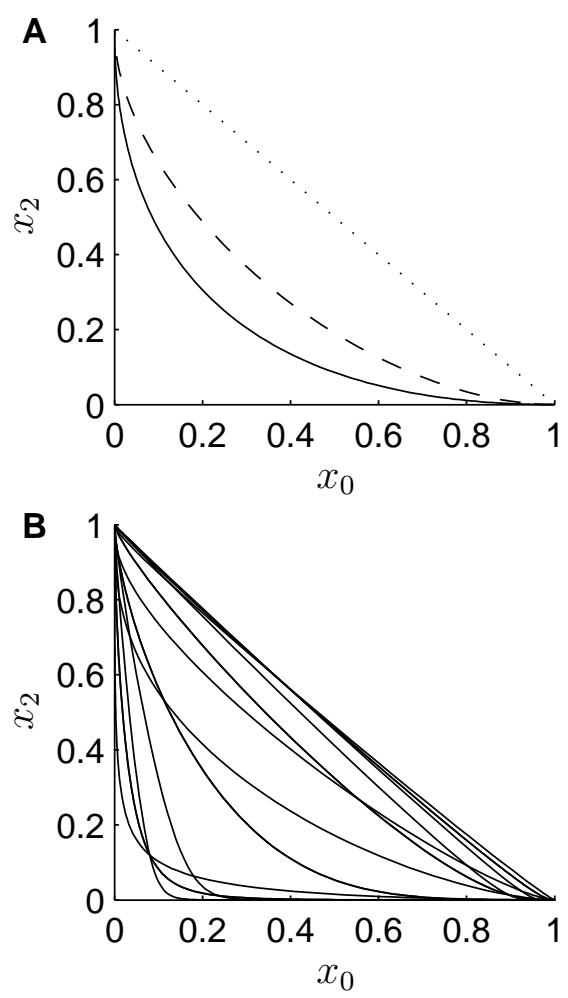

Figure 1: Curve of equilibrium points of (25). A: Hodgkin-Huxley type chain (solid line) and an identical rate chain (dashed line). B: Some of the various possible equilibrium curves for Case 3, simple exponential rate functions. 


\subsection{Eigenvectors of the Equilibrium}

System (25) will have two negative eigenvalues at the equilibrium point on $C$, and the relative magnitude of these two values along with the orientation of the corresponding eigenvectors will dictate the behaviour of solutions. As we stated earlier, we are interested in solutions whose initial value is some other point along the curve $C$. For the Hodgkin-Huxley system, the curve $C$ itself will be the solution, but not for the identical rate system. The solution to the Hodgkin-Huxley system will have a mixture of both eigenmodes but, as we show in this subsection, there are initial conditions for which the solution of the identical rate system is purely along the slow eigenvector, and thus exhibits a single exponential time course.

The eigenvalues of (25) at the equilibrium given by (26) are

$$
\lambda=\frac{1}{2}\left[-\left(r_{1}+l_{1}+r_{2}+l_{2}\right) \pm \sqrt{D}\right]
$$

where

$$
\begin{aligned}
D & =\left(r_{1}+l_{1}+r_{2}+l_{2}\right)^{2}-4\left(\left(r_{1}+l_{1}\right)\left(r_{2}+l_{2}\right)-l_{1} r_{2}\right) \\
& =\left(r_{1}+l_{1}+r_{2}+l_{2}\right)^{2}-4\left(r_{1} r_{2}+l_{1} l_{2}+r_{1} l_{2}\right) \\
& =\left(\left(r_{1}+l_{1}\right)-\left(r_{2}+l_{2}\right)\right)^{2}+4 l_{1} r_{2} .
\end{aligned}
$$

In (31), the plus sign is for the larger (less negative, slower motion) eigenvalue and the minus sign gives the smaller (more negative, faster motion) eigenvalue. As we already know, for the Hodgkin-Huxley type chain, Case 1, these eigenvalues are

$$
\lambda_{1}=-(\alpha+\beta), \quad \lambda_{2}=-2(\alpha+\beta),
$$

and for the identical rate chain, Case 2, they are

$$
\lambda_{1}=-(\alpha+\beta)+\sqrt{\alpha \beta}, \quad \lambda_{2}=-(\alpha+\beta)-\sqrt{\alpha \beta} .
$$

Thus, the IR chain has eigenvalues which are both smaller in magnitude than the HH type chain.

Since the corresponding eigenvectors, $v$, satisfy either

$$
-\left(r_{1}+l_{1}+\lambda\right) v_{1}-l_{1} v_{2}=0 \quad \text { or } \quad-r_{2} v_{1}-\left(l_{2}+r_{2}+\lambda\right) v_{2}=0
$$

they may be expressed as either

$$
v=\left[\begin{array}{c}
-l_{1} \\
\frac{1}{2}\left[\left(r_{1}+l_{1}\right)-\left(r_{2}+l_{2}\right) \pm \sqrt{D}\right]
\end{array}\right]
$$

or

$$
v=\left[\begin{array}{c}
\frac{1}{2}\left[\left(r_{2}+l_{2}\right)-\left(r_{1}+l_{1}\right) \pm \sqrt{D}\right] \\
-r_{2}
\end{array}\right] .
$$

From the third expression for $D$ in (32) it is then clear that the eigenvector corresponding to the slow motion has negative slope while the eigenvector corresponding to the fast motion has positive slope. It is therefore, impossible for the fast eigenvector to intersect the strictly decreasing curve $C$ at any place other than the equilibrium point. 


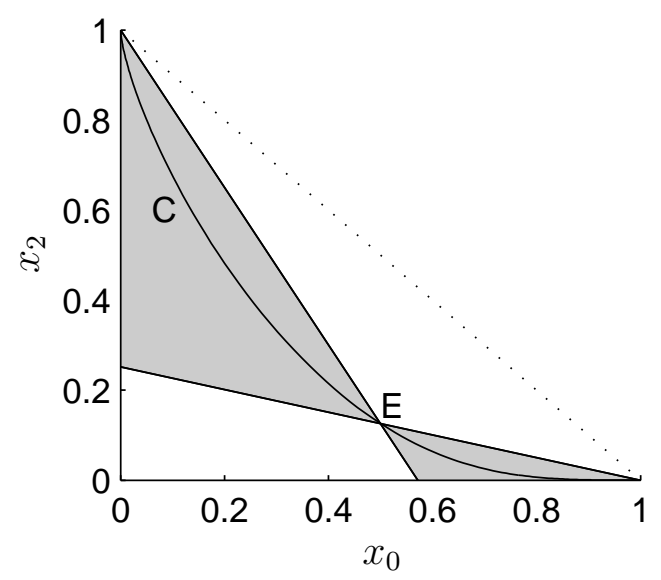

Figure 2: Slopes (shaded region) of the eigenvector of the equilibrium point $E$ which result in a second intersection with $C$.

Theorem 4 Under Assumptions 1 and 2, the eigenspace for the equilibrium (26) of (25) corresponding to the slow eigenvalue (31) either intersects the curve $C$ tangentially at the equilibrium, or it intersects in two places.

Proof: One intersection is at the equilibrium point, $E$, itself. Since the curve $C(V)$ is the graph of a strictly decreasing function on $\Delta$ whose second derivative does not change sign, and which goes through the points $(1,0)$ and $(0,1)$, it follows that if the slope $s_{s}$ of the slow eigenvector satisfies

$$
\frac{x_{2}-1}{x_{0}-0} \leq s_{s} \leq \frac{x_{2}-0}{x_{0}-1},
$$

where $x_{0}$ and $x_{2}$ are the coordinates of the equilibrium point $E$ given by (26), then there will be a second intersection with $C$ unless the slope of the eigenvector exactly matches the slope of the equilibrium curve, as illustrated in Fig. 2. This inequality simplifies to

$$
-\left(r_{1}+l_{1}\right) / l_{1} \leq s_{s} \leq-r_{2} /\left(r_{2}+l_{2}\right) .
$$

Using (33) for the slope of the eigenvector and the second expression for $D$ in (32), the left inequality in (35) becomes

$$
\left(r_{1}+l_{1}\right) \geq \frac{1}{2}\left[\left(r_{1}+l_{1}\right)-\left(r_{2}+l_{2}\right)+\sqrt{\left(r_{1}+l_{1}+r_{2}+l_{2}\right)^{2}-4\left(r_{1} r_{2}+l_{1} l_{2}+r_{1} l_{2}\right)}\right]
$$

which is equivalent to

$$
r_{1}+l_{1}+r_{2}+l_{2} \geq \sqrt{\left(r_{1}+l_{1}+r_{2}+l_{2}\right)^{2}-4\left(r_{1} r_{2}+l_{1} l_{2}+r_{1} l_{2}\right)},
$$

which clearly always holds. Similarly, using (34) for the slope, $s_{s}$, the right inequality in (35) becomes

$$
\left(\frac{1}{2}\left[\left(r_{2}+l_{2}\right)-\left(r_{1}+l_{1}\right)+\sqrt{\left(r_{1}+l_{1}+r_{2}+l_{2}\right)^{2}-4\left(r_{1} r_{2}+l_{1} l_{2}+r_{1} l_{2}\right)}\right]\right)^{-1} \geq 1 /\left(r_{2}+l_{2}\right)
$$


which also simplifies to (36).

We have already argued that the equilibrium curve $C(V)$ is itself a solution of the Hodgkin-Huxley system. (More precisely, for a fixed voltage level, $C$ is the union of the equilibrium point and two solutions given by (15)-(16) approaching the equilibrium from opposite sides.) Here, we verify that the slow eigenvector is in fact tangent to the equilibrium curve for the $\mathrm{HH}$ system. For this system, we have $r_{1}=2 r_{2}=2 \alpha$, and $2 l_{1}=l_{2}=2 \beta$. Thus, from (33) the slopes of the eigenvectors are

$$
\begin{aligned}
s & =-\frac{1}{2}\left[\left(\frac{2 \alpha}{\beta}+1\right)-\left(\frac{\alpha}{\beta}+2\right) \pm \sqrt{\left(\frac{2 \alpha}{\beta}+1+\frac{\alpha}{\beta}+2\right)^{2}-4\left(\frac{2 \alpha^{2}}{\beta^{2}}+2+\frac{4 \alpha}{\beta}\right)}\right] \\
& =-\frac{1}{2}\left[\frac{\alpha}{\beta}-1 \pm \sqrt{\left.9\left(\frac{\alpha}{\beta}+1\right)^{2}-8 \frac{\alpha^{2}}{\beta^{2}}-8-16 \frac{\alpha}{\beta}\right]}\right. \\
& =-\frac{1}{2}\left[\frac{\alpha}{\beta}-1 \pm \sqrt{\left(\frac{\alpha}{\beta}+1\right)^{2}}\right],
\end{aligned}
$$

and, therefore,

$$
s_{s}=-\frac{\alpha}{\beta}, \quad \text { and } \quad s_{f}=1 .
$$

Working from (29) and noting that $L=\frac{\beta}{2 \alpha}=\frac{1}{4 R}$ we obtain

$$
\frac{d x_{2}}{d x_{0}}=\frac{R^{\prime}\left(\frac{1}{4 R}+1\right)-R\left(\frac{-R^{\prime}}{4 R^{2}}\right)}{\left(\frac{-R^{\prime}}{4 R^{2}}\right)(R+1)-\frac{1}{4 R} R^{\prime}}=\frac{\frac{R^{\prime}}{4 R}(2+4 R)}{-\frac{R^{\prime}}{4 R}\left(2+\frac{1}{R}\right)}=-2 R=-\frac{\alpha}{\beta} .
$$

Similar computations for Case 2 , the identical rate chain, where $L=\frac{\beta}{\alpha}=\frac{1}{R}$, yield

$$
s_{s}=-\sqrt{\frac{\alpha}{\beta}}, \quad s_{f}=\sqrt{\frac{\alpha}{\beta}}, \quad \text { and } \quad \frac{d x_{2}}{d x_{0}}=-\frac{R(R+2)}{2 R+1}=-\frac{\alpha(\alpha+2 \beta)}{\beta(2 \alpha+\beta)} .
$$

A simple further calculation shows that the slope of the slow eigenvector is less than, equal to, or greater than, the tangent to the equilibrium curve $C$ if and only if $\alpha$ is less than, equal to, or greater than $\beta$, respectively. Since by Assumption 2, these two functions have opposite signed derivatives with respect to $V$, it follows that there will be one place on the equilibrium curve where the slow eigenvector is tangent to the curve. From (26), it follows that this point of tangency occurs when $x_{0}=x_{2}$. For equilibria on $C$ with $x_{2}>x_{0}$ (which from (26) dictates that $\alpha>\beta$ ), the slow eigenvector will have slope greater than the tangent to the curve and so will intersect $C$ to the right of the equilibrium. Conversely, the slow eigenvector emanating from equilibria on $C$ with $x_{2}<x_{0}$ will intersect $C$ to the left of the equilibrium. Thus, for every final voltage level (except the one at which $\alpha=\beta$ ), there is always some initial voltage level such that a voltage jump to the final level from the initial level applied to a system at equilibrium will result in a single exponential time course of the solution components $x_{0}$ and $x_{2}$ toward the new equilibrium point. This feature is a distinguishing feature for identical rate chains compared to Hodgkin-Huxley type chains. 


\subsection{Relative Sizes of the Solution Components}

It is informative to look at the relative sizes of the components corresponding to the two eigenvalues of the solution for $x_{2}$, comparing the Hodgkin-Huxley type chain with the identical rate chain. We assume that initially the system is maintained at a holding potential $W$, and that it is at equilibrium there. Thus the initial condition is given by (26) where the functions are evaluated at $W$. At time zero, the voltage is instantaneously changed to $V$ so that the globally attractive equilibrium point is given by (26) with the functions evaluated at $V$. We assume that $x_{2}$ is the only open state, and it is the only measurable quantity. (If one is measuring ionic currents only, then this is the case; however, information about a certain linear combination of the states $x_{i}$ can be obtained by measuring gating currents.) The solution for $x_{2}$ is of the form

$$
x_{2}(t)=a e^{\lambda_{1} t}+b e^{\lambda_{2} t}+c,
$$

where $\lambda_{1}$ is the slow eigenvalue and $\lambda_{2}$ the fast one. We are interested in the ratio $p=|b / a|$. The relevant equation to be solved is

$$
\left[\begin{array}{l}
x_{0}(W) \\
x_{2}(W)
\end{array}\right]=\left[\begin{array}{cc}
1 / s_{s} & 1 / s_{f} \\
1 & 1
\end{array}\right]\left[\begin{array}{l}
a \\
b
\end{array}\right]+\left[\begin{array}{l}
x_{0}(V) \\
x_{2}(V)
\end{array}\right] .
$$

Solving the above for $a$ and $b$, we obtain

$$
\left[\begin{array}{l}
a \\
b
\end{array}\right]=\frac{1}{s_{s}-s_{f}}\left[\begin{array}{cc}
-s_{f} s_{s} & s_{s} \\
s_{f} s_{s} & -s_{f}
\end{array}\right]\left[\begin{array}{l}
x_{0}(W)-x_{0}(V) \\
x_{2}(W)-x_{2}(V)
\end{array}\right]
$$

thus,

$$
p=\left|\frac{b}{a}\right|=\left|\frac{\left(x_{0}(W)-x_{0}(V)\right)-\frac{1}{s_{s}}\left(x_{2}(W)-x_{2}(V)\right)}{\left(x_{0}(W)-x_{0}(V)\right)-\frac{1}{s_{f}}\left(x_{2}(W)-x_{2}(V)\right)}\right| .
$$

For an HH type chain, Case 1, after some algebra, the ratio $p$ becomes

$$
p_{H H}=\frac{|Q(V)-Q(W)|}{2 Q(V)(1+Q(W))},
$$

where $Q=\alpha / \beta$. For the IR chain, Case 2 , the ratio is

$$
p_{I R}=\left|\frac{(Q(V)+Q(W)+1)(\sqrt{Q(V)}-1)-Q(V) Q(W)+1}{(Q(V)+Q(W)+1)(\sqrt{Q(V)}+1)+Q(V) Q(W)-1}\right| .
$$

For both Case 1 and Case 2, the ratio $x_{2} / x_{0}$ at equilibrium is given by $Q^{2}$. Plots of the surfaces $p$ for both cases as a function of $x_{2}(V) / x_{0}(V)$ and $x_{2}(W) / x_{0}(W)$ (on a log scale) are shown in Fig. 3. Note that for the Hodgkin-Huxley type chain, plotted in Fig. 3A, the fast component approaches zero along the diagonal since the slow eigenvector is tangent to the equilibrium curve and the start and end points are moving together along this curve as the diagonal is approached. When the end ratio $x_{2}(V) / x_{0}(V)$ is larger than the initial ratio 

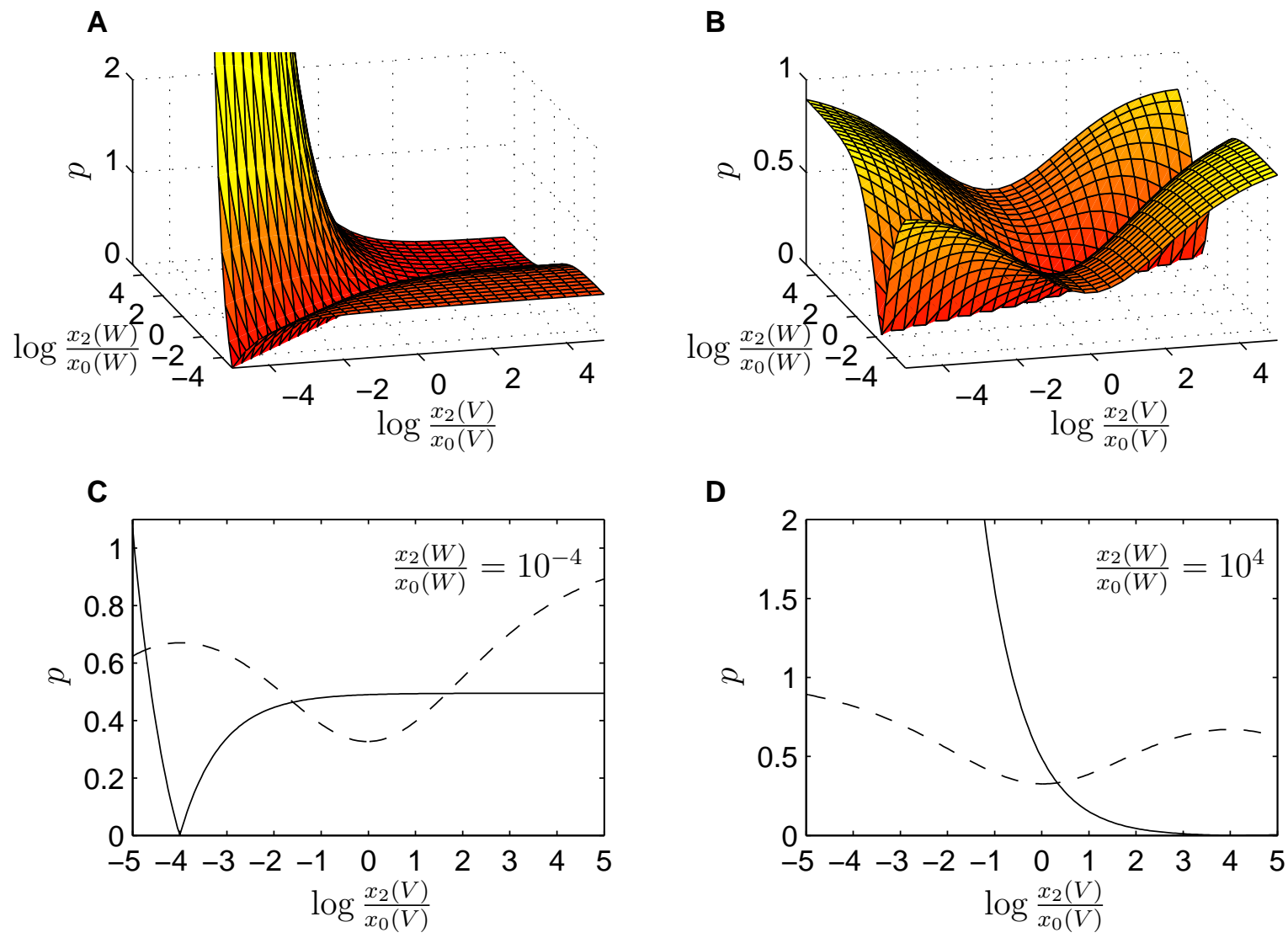

Figure 3: The ratio $p$ of the coefficients of the fast component to the slow component in the solution $x_{2}(t)$ as a function of the ratios $x_{2} / x_{0}$ at the initial voltage, $W$, and the final voltage, $V$. A: The Hodgkin-Huxley type chain. B: The identical rate chain. C: Cross section of both surfaces in A and B through $x_{2}(W) / x_{0}(W)=10^{-4}$. Hodgkin-Huxley surface (from A) is the solid line; dashed line is the identical rate surface from B. D: Cross section through $x_{2}(W) / x_{0}(W)=10^{4}$. Lines as in C. Note that vertical scales differ on these plots. 
$x_{2}(W) / x_{0}(W)$, the fast component remains less than half the slow component, however, when the end ratio is smaller than the initial ratio, the fast component can become significantly larger than the slow component. In contrast, the identical rate chain, plotted in Fig. 3B, always has the fast component of the solution smaller than the slow component, and except where the initial and end points are both at extreme ends of the equilibrium curve, the fast component is less than half the size of the small component. In addition, there is a line of points where the fast component is zero, that is, the solution contains a slow component only, corresponding to where the slow eigenvector meets the equilibrium curve at the start location.

Two typical voltage-clamp experimental situations are plotted in Figs. 3C and D. We discuss them in terms of an activation gate where $x_{2} / x_{0}$ is an increasing function of voltage, although an inactivation gate with $x_{2} / x_{0}$ decreasing works analogously. A large ionic current can be generated by holding the channel at a very low potential $W$ and jumping up to a very high potential $V$. Typical cells can tolerate voltage jumps on the order of $100 \mathrm{mV}$. Using typical rate functions, such a jump can correspond to equilibrium ratios $x_{2} / x_{0}$ differing from five to fifteen orders of magnitude. For example, the classical $\mathrm{HH}$ rate constants for the sodium channel activation gate give $x_{2}(-60) / x_{0}(-60)=2.4 \times 10^{-10}$ and $x_{2}(40) / x_{0}(40)=$ 19.8 (Hodgkin and Huxley, 1952). For illustration, assume $W$ is sufficiently small so that $x_{2}(W) / x_{0}(W)=10^{-4}$, then Fig. 3C shows the relative size of the fast component to the slow component for the elicited current as a function of the end ratio $x_{2}(V) / x_{0}(V)$. For the $\mathrm{HH}$ model, the size of the fast component quickly rises to half the size of the slow component, so that virtually all except the smallest voltage jumps will yield ionic current traces where the slow component is twice the size of the fast component. For the IR model, the fast component varies non-monotonically between about 0.4 and 1 . For large voltage steps, the fast component will be comparable to the size of the slow component. The second voltage clamp situation is where the system is initially held at a high voltage $W$ and then dropped instantaneously to a low value $V$. The channel is thus initially open and then closes. This corresponds to measuring tail currents, and the relative sizes of the fast and slow components are shown in Fig. 3D, where for illustration we have chosen $x_{2}(W) / x_{0}(W)=10^{4}$. Note that here the IR chain again has the fast component generally between 0.5 and 1 times the size of the slow component. In contrast, the $\mathrm{HH}$ type chain shows a rapid rise in the relative size of the fast component as the end point ratio $x_{2}(V) / x_{0}(V)$ becomes small. This is a distinguishing feature between Hodgkin-Huxley models and identical rate models (at least for this three-state system). We conjecture that it remains a distinguishing feature when more states are considered. Specifically, tail currents (the decaying ionic current measured when the channel moves from a fully open state to a fully closed one) for $\mathrm{HH}$ models are composed primarily of a fast decaying component corresponding to the most negative eigenvalue while tail currents for IR models are composed of a relatively even mix of components distributed across all the negative eigenvalues. The first part of this conjecture is obviously true when one considers Eqs. (15) and (17). These yield

$$
x_{n}=m^{n}=\sum_{j=0}^{n}\left(\begin{array}{l}
n \\
j
\end{array}\right) m_{\infty}^{n-j}\left(m_{0}-m_{\infty}\right)^{j} e^{-j(\alpha+\beta) t},
$$


so that the ratio of the component of $x_{n}$ corresponding to the most negative eigenvalue, $-n(\alpha+\beta)$, to the component corresponding to the eigenvalue $-j(\alpha+\beta), 1 \leq j<n$, is

$$
\frac{\left(m_{0}-m_{\infty}\right)^{n}}{\left(\begin{array}{c}
n \\
j
\end{array}\right)\left(m_{0}-m_{\infty}\right)^{j} m_{\infty}^{n-j}}=\frac{1}{\left(\begin{array}{c}
n \\
j
\end{array}\right)}\left(\frac{m_{0}}{m_{\infty}}-1\right)^{n-j} .
$$

Therefore, as $m_{\infty} \rightarrow 0$ (which implies $x_{n}(V) / x_{0}(V) \rightarrow 0$ ), this ratio is unbounded.

\section{Discussion}

When developing multi-state models of ion channels constructed of several (typically four) membrane spanning subunits, it has been commonly assumed that the symmetry of the physical channel should be reflected in some symmetry of the model. If each subunit can have a "closed" and an "open" state, then four subunits generate five different states for the channel (zero through four subunits "open"). For this reason, chains of five states with the last state open, or leading to subsequent states from which the channel opens are common (Bezanilla, 2000). In order to reduce the number of free parameters in the models, these chains are often constructed as Hodgkin-Huxley type chains or identical rate chains. Our geometric analysis of the three-state chains suggests that Hodgkin-Huxley type chains and identical rate chains are distinguishable in their movement from a fully open to a fully closed state. Currents of the former type decay primarily through the fastest mode (most negative eigenvalue) while currents of the latter appear to decay with a relatively even mix of fast and slower modes. As has been observed by others, for example Zagotta et al. (1994a), tail currents for some channels display a nearly single exponential time course of deactivation. This suggests an $\mathrm{HH}$ type chain in the activation pathway, however, models of specific ion channels often involve inactivation or passage from the open state to states not directly in the activation pathway (Zagotta et al., 1994b; Bezanilla, 2000). These additional states have the potential of significantly altering the kinetics of the open state. Nonetheless, an understanding of differences in the single chain systems we have described here is an aid in understanding the behaviour of more complicated models.

We have concentrated on single chain models, although the first results of Sect. 2 are applicable to all multi-state models. If a channel also undergoes inactivation, then it can be modelled as a single chain system with additional closed states beyond the open state. In other words, the chain has $n+1$ states but the open state is not the last one, rather one in the middle. Some of our results are applicable to these models, but usually such chains are not $\mathrm{HH}$ type or IR chains, or not completely so. More commonly, inactivation involves the addition of states which generate potential loops in the network. For example, a multi-state model that is equivalent to the classical Hodgkin-Huxley model for the sodium conductance in the squid giant axon is

$\begin{array}{ccccccccc}x_{0} & \stackrel{4 \alpha_{m}}{\rightleftarrows} & x_{1} & \underset{2 \alpha_{m}}{\stackrel{3 \alpha_{m}}{\rightleftarrows}} & x_{2} & \underset{3 \beta_{m}}{\stackrel{2 \alpha_{m}}{\rightleftarrows}} & x_{3} & \underset{4 \beta_{m}}{\stackrel{\alpha_{m}}{\rightleftarrows}} & x_{4} \\ \alpha_{h} \uparrow \downarrow \beta_{h} & & \alpha_{h} \uparrow \downarrow \beta_{h} & & \alpha_{h} \uparrow \downarrow \beta_{h} & & \alpha_{h} \uparrow \downarrow \beta_{h} & & \alpha_{h} \uparrow \downarrow \beta_{h} \\ x_{5} & \stackrel{4 \alpha_{m}}{\rightleftarrows} & x_{6} & \underset{2 \alpha_{m}}{\rightleftarrows} & x_{7} & \underset{3 \beta_{m}}{\stackrel{2 \alpha_{m}}{\rightleftarrows}} & x_{8} & \underset{3 \beta_{m}}{\stackrel{\alpha_{m}}{\rightleftarrows}} & x_{9}\end{array}$


where $x_{4}$ is the open state and the $m$ and $h$ subscripts refer to the activation and inactivation rate functions. Other models including inactivation tend to have less symmetry than the above model but also introduce loop connections between a number of states (Bezanilla, 2000; Zagotta et al., 1994b). More work needs to be done on such models to get a fundamental understanding of their behaviour.

In the three-state IR model, we showed that (except in the case where the final condition is $x_{0}=x_{2}$ ) there is always an intersection of the slow eigenvector with the curve of equilibria. This means that in the typical situation, where the initial condition lies on the equilibrium curve and a sudden change in the voltage is applied, for each final voltage level there will always be a particular initial voltage level such that the solution for this case is a single exponential corresponding to the slow eigenvalue. This is another distinguishing feature between the three-state identical rate chain and the three state Hodgkin-Huxley chain; the $\mathrm{HH}$ chain always has multi-exponential solutions involving all the eigenvalues. In a higher dimensional setting, the generalization of this result is not immediately clear. Geometrically, the equilibrium curve and the eigenspace for the slow eigenvector are one-dimensional manifolds and so their meeting in a space of higher than two dimensions is not expected. Thus, even for a four-state IR model a single exponential solution connecting two points on the equilibrium curve is not likely. However, it is not clear whether the structure of the problem with the symmetries of the IR chain might not dictate that the eigenspace must intersect the equilibrium curve. More work in this direction is required.

\section{Acknowledgements}

This research was partly supported by the Natural Sciences and Engineering Research Council of Canada. D.N. acknowledges the support of the University of Guelph through an Undergraduate Research Assistantship.

\section{References}

Bähring, R., Boland, L. M., Varghese, A., Gebauer, M., Pongs, O., 2001. Kinetic analysis of open- and closed-state inactivation transitions in human Kv4.2 A-type potassium channels. J. Physiol. 535, 65-81.

Ball, F. G., Rice, J. A., 1992. Stochastic models for ion channels: Introduction and bibliography. Math. Biosci. 112, 189-206.

Bezanilla, F., 2000. The voltage sensor in voltage-dependent ion channels. Physiol. Rev. 80, $555-592$.

Chanda, B., Bezanilla, F., 2002. Tracking voltage-dependent conformational changes in skeletal muscle sodium channel during activation. J. Gen. Physiol. 120, 629-645.

Destexhe, A., Huguenard, J. R., 2000. Nonlinear thermodynamic models of voltagedependent currents. J. Comp. Neurosci. 9, 259-270. 
Destexhe, A., Mainen, Z. F., Sejnowski, T. J., 1994. Synthesis of models for excitable membranes, synaptic transmission and neuromodulation using a common kinetic formalism. J. Comp. Neurosci. 1, 195-230.

Eyring, H., Lumry, R., Woodbury, J. W., 1949. Some applications of modern rate theory to physiological systems. Record Chem. Prog. 10, 100-114.

Fitzhugh, R., 1965. A kinetic model of the conductance changes in nerve membrane. J. Cell. Comp. Physiol. 66, 111-115.

Fredkin, D. R., Montal, M., Rice, J. A., 1985. Identification of aggregated Markovian models: Application to the nicotinic acetylcholine receptor. In: Cam, L. M. L., Olshen, R. A. (Eds.), Proceedings of the Berkeley Conference in Honor of Jerzy Neyman and Jack Kiefer. Vol. 1. Institute of Mathematical Statistics, Wadsworth Advanced Books \& Software, Monterey, CA, pp. 269-289.

Hill, T. L., Chen, Y. D., 1972. On the theory of ion transport across nerve membranes. VI. free energy and activation free energies of conformational change. Proc. Natl. Acad. Sci. USA $69,1723-1726$.

Hille, B., 1992. Ionic Channels of Excitable Membranes. Sinauer, Sunderland, MA.

Hodgkin, A. L., Huxley, A. F., 1952. A quantitative description of membrane current and its application to conduction and excitation in nerve. J. Physiol. Lond. 117, 500-544.

Kargol, A., Smith, B., Millonas, M. M., 2002. Applications of nonequilibrium response spectroscopy to the study of channel gating. Experimental design and optimization. J. Theor. Biol. 218, 239-258.

Kienker, P., 1989. Equivalence of aggregated Markov models of ion-channel gating. Proc. Royal Soc. London B 236, 269-309.

Kijima, H., Kijima, S., 1997. Theoretical approaches to ion channel dynamics and the firstorder reaction. Prog. Cell Res. 6, 295-304.

Levitt, D. G., 1989. Continuum model of voltage-dependent gating. Biophys. J. 55, 489-498.

Liebovitch, L. S., Fischbarg, J., Koniarek, J. P., 1987. Ion channel kinetics: A model based on fractal scaling rather than multistate Markov processes. Math. Biosci. 84, 37-68.

Liebovitch, L. S., Scheurle, D., Rusek, M., Zochowski, M., 2001. Fractal methods to analyze ion channel kinetics. Methods 24, 359-375.

Liebovitch, L. S., Todorov, A. T., 1996. Using fractals and nonlinear dynamics to determine the physical properties of ion channel proteins. Crit. Rev. in Neurobiol. 10, 169-187.

Mirsky, L., 1963. An Introduction to Linear Algebra. Oxford, Clarendon Press, London. 
Piper, D. R., Varghese, A., Sanguinetti, M. C., Tristani-Firouzi, M., 2003. Gating currents associated with intramembrane charge displacement in HERG potassium channels. PNAS 100, 10534-10539.

Roux, M. J., Olcese, R., Toro, L., Bezanilla, F., Stefani, E., 1998. Fast inactivation in Shaker $\mathrm{K}^{+}$channels. J. Gen. Physiol. 111, 625-638.

Tsien, R. W., Noble, D., 1969. A transition state theory approach to the kinetics of conductances in excitable membranes. J. Membr. Biol. 1, 248-273.

Yueh, W.-C., 2005. Eigenvalues of several tridiagonal matrices. Appl. Math. E-Notes 5, 6674 .

Zagotta, W. N., Hoshi, T., Dittman, J., Aldrich, R. W., 1994a. Shaker potassium channel gating II: Transitions in the activation pathway. J. Gen. Physiol. 103, 279-319.

Zagotta, W. N., Hoshi, T., Dittman, J., Aldrich, R. W., 1994b. Shaker potassium channel gating III: Evaluation of kinetic models for activation. J. Gen. Physiol. 103, 321-362. 